\title{
Rhino-orbital mucormycosis in COVID-19 patients-a new threat?
}

\author{
Sandeep Singh Awal ${ }^{1 *}$ (D) Som Subhro Biswas ${ }^{2}$ and Sampreet Kaur Awal ${ }^{3}$
}

\begin{abstract}
Background: Coronavirus disease 2019 (COVID-19) is known to be associated with a myriad of viral, fungal, and bacterial co-infections. Rhino-orbital mucormycosis is a rare angio-invasive fungal infection which has shown a rising trend in the setting of COVID-19.

Case presentation: We describe the imaging findings in 3 cases of rhino-orbital mucormycosis in patients with history of COVID-19. All cases had varying involvement of paranasal sinuses extending into the orbital compartment while case 3 had intracranial extension of infection.

Conclusions: Rhino-orbital mucormycosis can have aggressive necrosis of the involved paranasal sinuses and orbits with or without cerebral extension. Hence, the correct diagnosis is imperative as prompt antifungal drugs and surgical debridement can significantly reduce mortality and morbidity.
\end{abstract}

Keywords: COVID-19, Mucormycosis, Black turbinate sign, MRI, Case report

\section{Background}

The 2019 novel coronavirus disease (COVID-19) is an infectious viral disease caused by the severe acute respiratory syndrome coronavirus 2 (SARS-CoV-2) [1]. The ongoing COVID-19 outbreak has spread worldwide since the first human case was detected in the month of December 2019 [2, 3]. COVID-19 is known to cause respiratory symptoms primarily, ranging from mild to severe pneumonia [4]. However, it can be associated with a broad-spectrum of bacterial and fungal co-infections [5]. The current second wave of the COVID-19 pandemic in India has seen a rise in the rhino-orbital mucormycosis co-infections in COVID-19 patients [6].

Rhino-orbital mucormycosis is a rare invasive fungal infection that originates in the paranasal sinuses and may frequently extend into the orbits and cerebral parenchyma [7]. Uncontrolled diabetes mellitus and the use of corticosteroids for the treatment of respiratory symptoms are possible etiological factors [6, 7]. Mucormycosis can be life threatening as it has a high mortality rate

\footnotetext{
* Correspondence: jonty3awal@gmail.com

${ }^{1}$ Department of Radiology, Jeevandeep Diagnostics, Jamshedpur, India

Full list of author information is available at the end of the article
}

of over 50\% [8]. Early diagnosis, delineating the extent of the spread of infection, is necessary as medical and surgical intervention can reduce mortality and morbidity [7]. Hence, it is crucial for all radiology departments to be familiar with the imaging features of rhino-orbital mucormycosis.

We present the imaging findings in 3 cases of rhinoorbital mucormycosis co-infections in COVID-19 patients. Contrast-enhanced MRI (CE-MRI) protocol of the paranasal sinuses, brain, and orbits included axial T1, T2, T2 FLAIR, GRE, DWI, T2 FS, T1 FS post-contrast $(3 \mathrm{~mm}$ thickness), sagittal T2, T1 FS post-contrast ( $3 \mathrm{~mm}$ thickness), coronal T2, and T1 FS post-contrast ( $3 \mathrm{~mm}$ thickness) sequences. High-resolution CT thorax (HRCT thorax) of case 1 was done using 32 slice multidetector CT machine using thin sections (1 $\mathrm{mm}$ slice thickness). Histopathological evaluation of nasal discharge of all 3 cases was done on potassium hydroxide $(\mathrm{KOH})$ wet mount and further confirmed on culture using lactophenol cotton blue (LPCB) stain. Final diagnosis of mucormycosis was made based on clinical details, imaging findings, and histopathology. 


\section{Case presentation}

\section{Case 1}

A 65-year-old diabetic female presented with respiratory distress, left orbital pain, left-sided ptosis, nasal congestion and discharge, and fever of short duration. Reverse transcriptase-polymerase chain reaction (RTPCR) from nasopharyngeal swab for COVID-19 was positive.

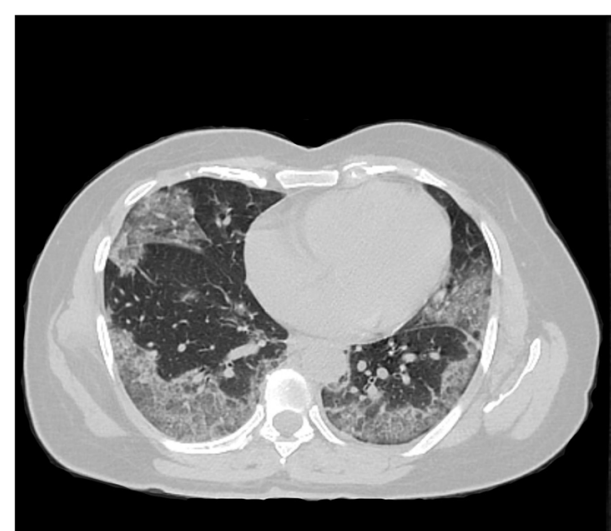

$\mathbf{A}$

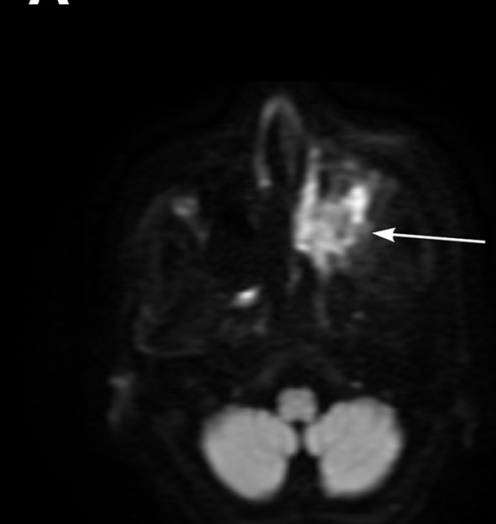

C

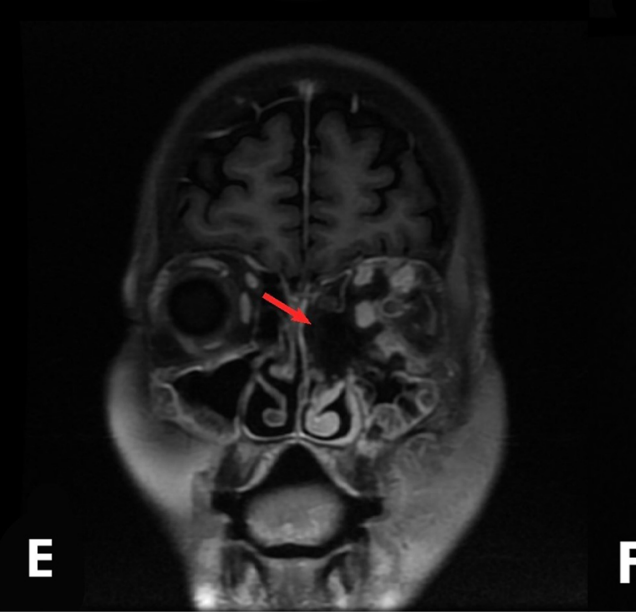

B

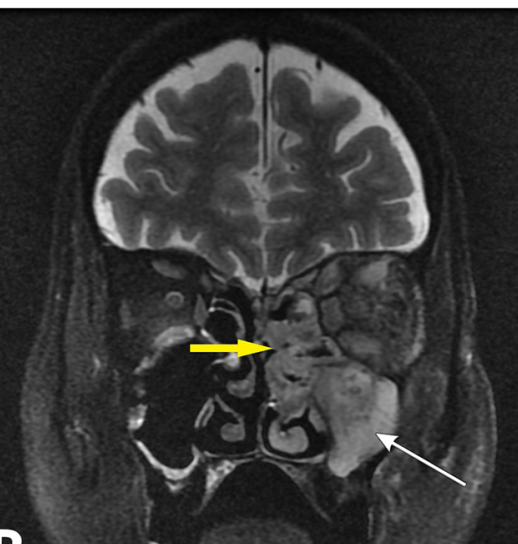

D
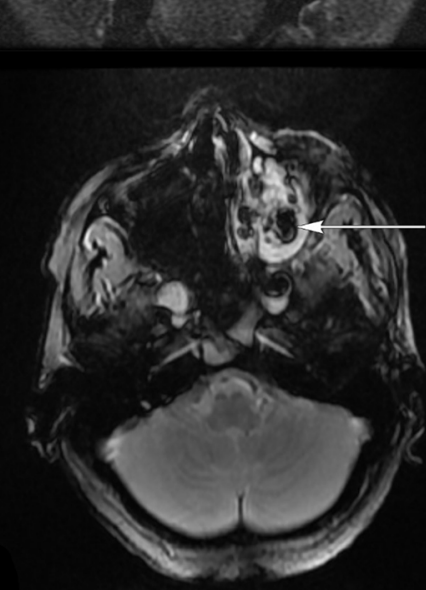

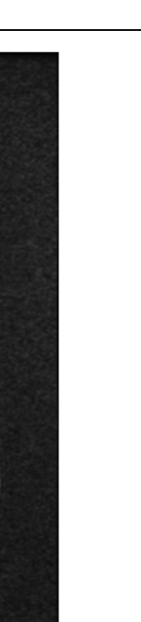

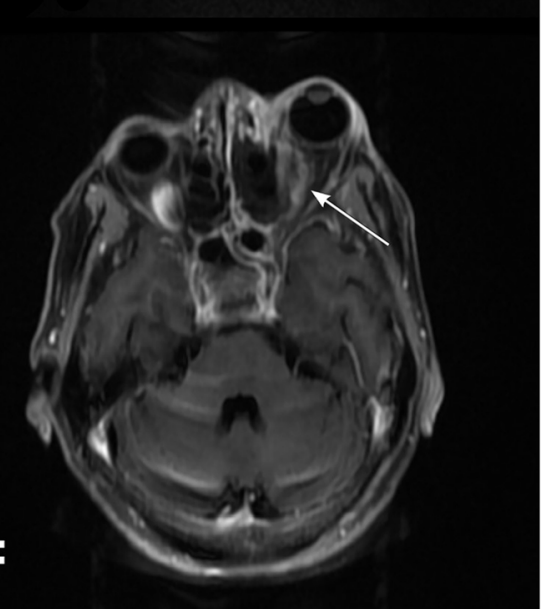

Fig. 1 a Axial HRCT thorax image showing ground-glass opacities in subpleural regions of bilateral lung parenchyma with "crazy paving appearance." b Coronal T2 FS image showing mucosal thickening and collection in the left maxillary sinus (white arrow), left ethmoidal sinus, and left middle nasal turbinate (yellow arrow) with inflamed extraocular muscles. c Axial DWI image showing restricted diffusion in the left maxillary sinus and left middle nasal turbinate. $\mathbf{d}$ Axial GRE image showing foci of blooming in the left maxillary sinus (white arrow). e Coronal T1 postcontrast image showing area of non-enhancing soft tissue in left middle nasal turbinate and within the left maxillary antrum ("black turbinate sign"). f Axial T1 post-contrast image showing enhancement and inflammation involving extraocular muscles of left orbit causing proptosis 
HRCT thorax (Fig. 1a) revealed multiple peripheral ground glass opacities in posterior subpleural regions of bilateral lung parenchyma with interlobular septal thickening (crazy paving appearance) [9].

CE-MRI of the paranasal sinuses and orbits revealed mucosal thickening and collection in all paranasal sinuses, predominantly in left maxillary (Fig. 1b), sphenoidal, and ethmoidal sinuses. Restricted diffusion on DWI (Fig. 1c) and blooming on GRE (Fig. 1d) were seen involving left middle nasal turbinate and left maxillary sinus. Post-contrast T1weighted images (Fig. 1e) showed enhancement in the involved structures with area of non-enhancing soft tissue in left middle nasal turbinate and within the left maxillary antrum ("black turbinate sign") $[10,11]$. Retroorbital fat, extraocular muscles of left orbit showed enhancement and inflammation on post-contrast $\mathrm{T} 1$ images with left sided proptosis (Fig. 1f).

Histopathological evaluation (HPE) of the nasal discharge revealed broad aseptate ribbon-like fungal hyphae on $\mathrm{KOH}$ wet mount [12]. Lactophenol cotton blue (LPCB) stain after $72 \mathrm{~h}$ of culture on Sabouraud dextrose agar (SDA) revealed broad aseptate ribbonlike hyphae branching at right angles with sporangium (Fig. 2).

\section{Case 2}

A 45-year-old female presented with right hemifacial pain and right orbital swelling for 5 days. Patient had past history of severe COVID-19 pneumonia for which she was hospitalized 3 weeks ago. She was treated with remdesivir, oxygen support, and intravenous methylprednisolone.

CE-MRI of the paranasal sinuses and orbits revealed mucosal thickening and collection in the right maxillary sinus causing blockage of right osteomeatal unit (Fig. 3a). Extension of inflammation with heterogenous post-contrast peripheral enhancement was seen in the right inferior orbital wall (Fig. 3b). Hypertrophied right middle and inferior nasal turbinates. Soft tissue swelling involving the right premaxillary soft tissue was seen (Fig. 3c). Mucosal thickening also noted in the ethmoidal and left maxillary sinus (Fig. 3b). $\mathrm{KOH}$ mount of nasal discharge revealed broad aseptate fungal hyphae, later confirmed on culture showing broad ribbon-like hyphae with sporangium (Fig. 3d).

\section{Case 3}

A 36-year-old male with recent history of COVID-19 pneumonia presented with persisting left hemifacial pain and rhinorrhea.

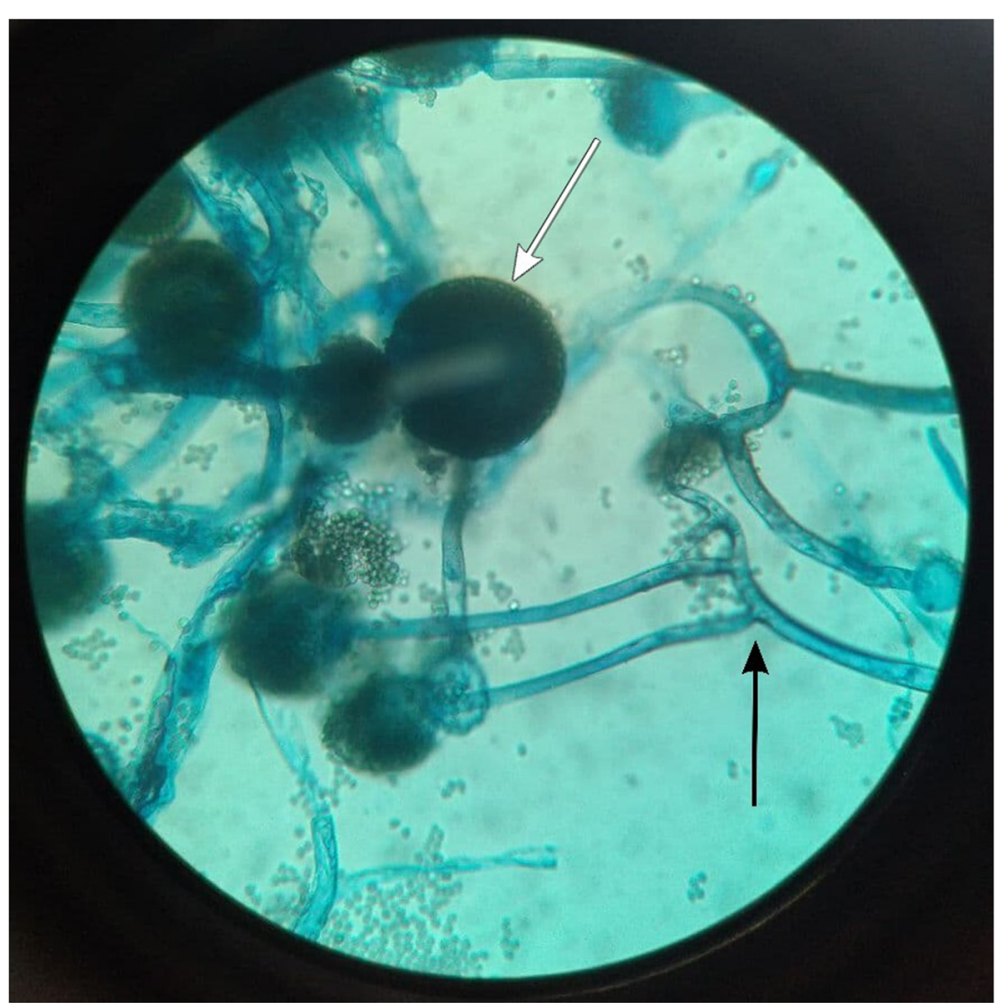

Fig. 2 Lactophenol cotton blue (LPCB) stain after culture on Sabouraud dextrose agar (SDA) showing broad aseptate ribbon-like hyphae branching at right angles (black arrow) with sporangium (white arrow) 


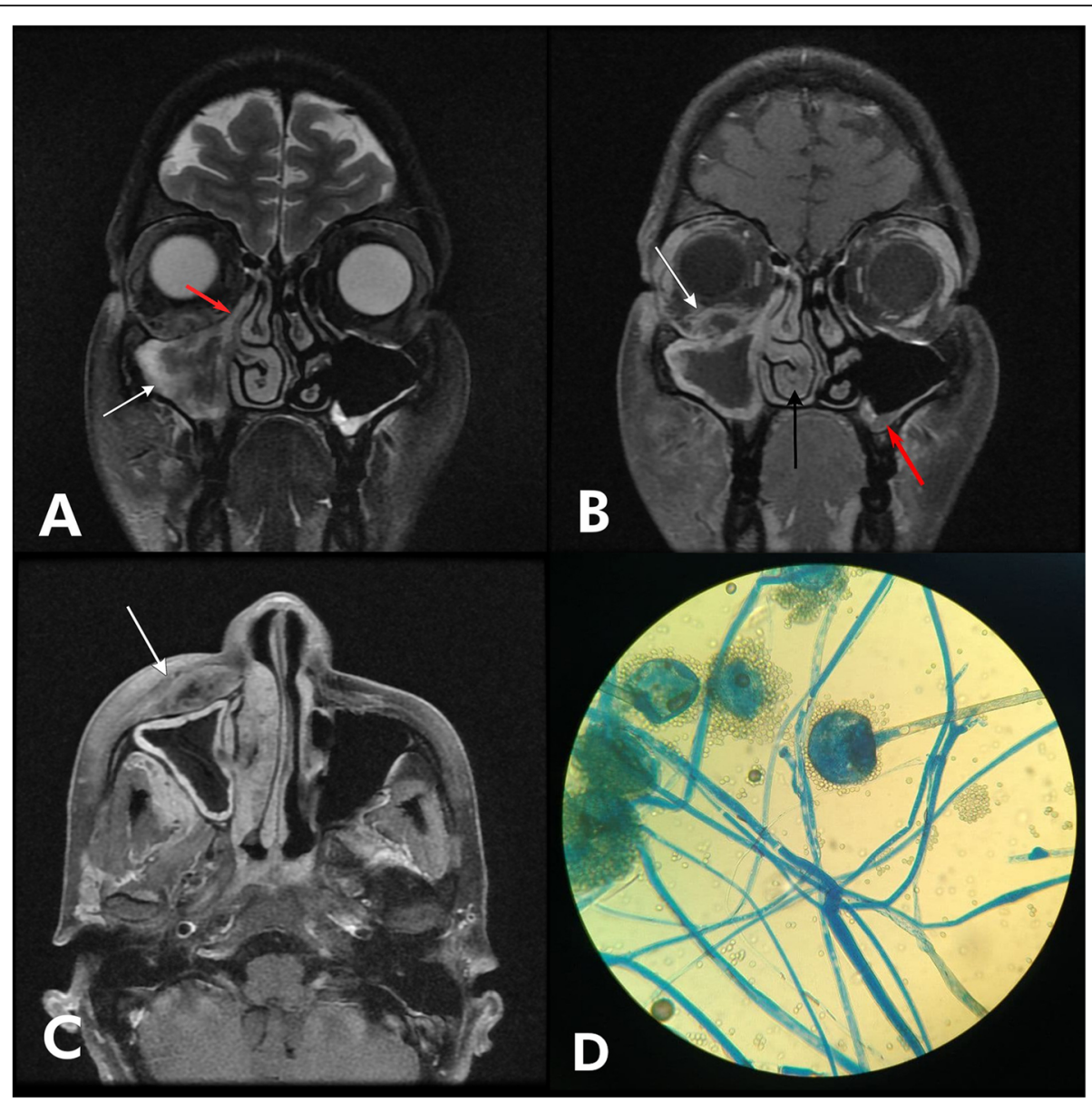

Fig. 3 a Coronal T2 image showing mucosal thickening in right maxillary sinus (white arrow) causing blockage of the right osteomeatal unit (red arrow). b Coronal T1 FS post-contrast image showing extension of inflammation in right inferior orbit (white arrow) with post-contrast peripheral enhancement, right inferior turbinate hypertrophy (black arrow), and left maxillary wall thickening (white arrow). c Axial T1 FS post-contrast image showing right pre-maxillary soft tissue swelling with enhancement (white arrow). $\mathbf{d}$ Lactophenol cotton blue (LPCB) stain showing broad ribbonlike fungal hyphae with sporangium

CE-MRI of the paranasal sinuses, brain, and orbits revealed mucosal thickening and collection involving frontal, ethmoidal, sphenoidal, and left maxillary sinuses (Fig. 4a). Left pre-maxillary soft tissue swelling was seen. Bony defects involving inferior orbital wall was seen with extension of soft tissue component into left inferomedial orbit (Fig. 4b). Small, shrunken left eye globe was seen suggestive of phthisis bulbi [13]. Enhancement involving left infratemporal fossa region and left medial temporal lobe was noted, suggestive of intracranial extension (Fig. 4c).

$\mathrm{KOH}$ wet mount revealed broad non septate fungal hyphae. Broad aseptate ribbon-like hyphae with sporangium is seen on the LPCB stain after $72 \mathrm{~h}$ of culture in SDA agar (Fig. 4d).

\section{Discussion}

A myriad of co-infections of viral, fungal, and bacterial etiology and associated complications have been encountered in patients of COVID-19 [14, 15]. During the current second wave of the COVID-19 pandemic in India, an increase in fungal infections, predominantly rhino-orbital mucormycosis, has been documented [6].

Mucormycosis is a rare, opportunistic fungal infection which causes angio-invasive disease leading to aggressive necrosis and infarction of the involved tissues. Rhinoorbital mucormycosis involves the paranasal sinuses and orbits and may extend into the cerebral parenchyma [7, 15]. Underlying predisposing factors include uncontrolled diabetes mellitus, immunocompromised status, systemic use of corticosteroids, pre-existing respiratory pathology, cancer, and stem cell transplant $[5,7,15]$. Among these, diabetes is one of the most common etiological factors [16].

MRI is a valuable modality that can be used to diagnose mucormycosis infections involving sino-nasal region, orbits, and possible intracranial extension [17]. The multiplanar capabilities of MRI with its superior soft tissue 


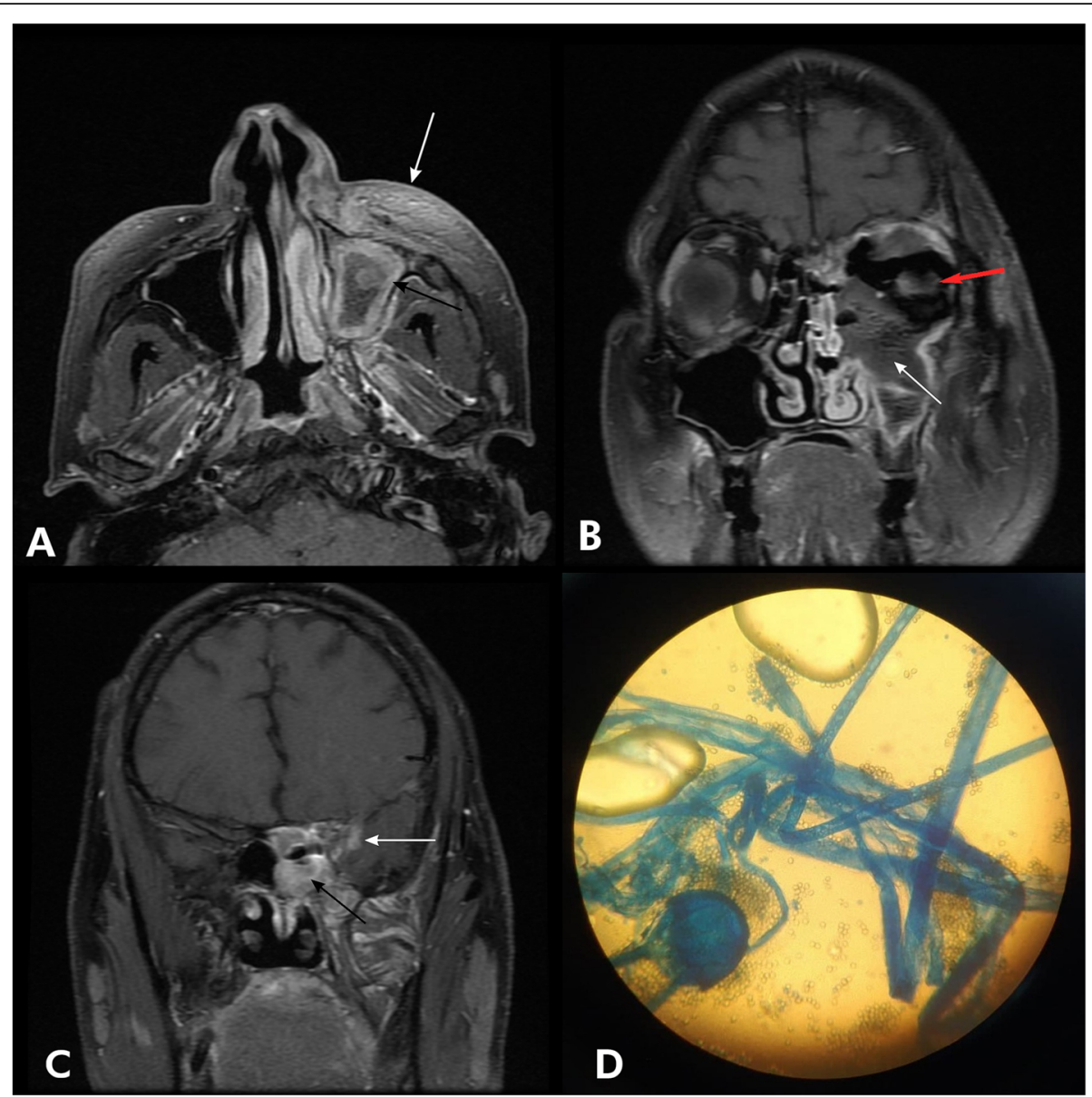

Fig. 4 a Axial T1 post-contrast image showing mucosal thickening and collection involving left maxillary sinus (black arrow) with pre-maxillary soft tissue swelling (white arrow). b Axial T1 post-contrast image showing inferior orbital wall with extension of soft tissue component into left inferomedial orbit (white arrow) and left phthisis bulbi (red arrow). c Coronal T1 post-contrast image showing left sphenoid sinusitis (black arrow) with an area of enhancement involving left medial temporal lobe (white arrow). $\mathbf{d}$ Lactophenol cotton blue (LPCB) stain showing broad aseptate ribbon-like hyphae with sporangium

depiction are helpful in delineating the anatomical extent of disease as well as its complications $[17,18]$.

Imaging findings of mucormycosis include mucosal thickening and/or opacification of the involved paranasal sinuses. Majority of the lesions appear hypointense on T1-weighted images and variable to hyperintense on T2weighted images [17]. Low signal intensity of fungal elements on T2-weighted images along with restricted diffusion on DWI may be seen [11]. Hypertrophy of nasal turbinates with nasal secretions is seen with nasal involvement [18]. Post-contrast enhancement can be seen in the thickened mucosa and involved tissues. However, areas of non-enhancing soft tissue may be seen within the affected turbinates and/or paranasal sinuses, known as the "black turbinate sign" $[10,11]$. This sign may help in the early detection of nasal mucormycosis [11].

Extrasinus extension into orbital compartment, face is commonly encountered and may further extend into the infratemporal fossa, cavernous sinus, skull base, and intracranial compartment [18]. Post-treatment follow-up MRI may be needed in some cases.

\section{Conclusions}

Imaging plays a key role in the early identification of rhino-orbital mucormycosis and delineating the extent of infection. Prompt diagnosis and treatment of rhinoorbital mucormycosis is the "sine qua non" as antifungal drugs and surgical debridement can successfully control the infection and thus reduce the high mortality and morbidity associated with mucormycosis. Due to the changing trends in the COVID-19 pandemic, it is an absolute necessity for all radiologists to be well aware of the imaging features of rhino-orbital mucormycosis and its possible complications.

\section{Abbreviations}

COVID-19: Coronavirus disease 2019; SARS-CoV-2: Severe acute respiratory syndrome coronavirus 2; RI: Magnetic resonance imaging; CE-MRI: Contrastenhanced MRI; FLAIR: Fluid-attenuated inversion recovery; GRE: Gradient 
recalled echo; DWI: Diffusion-weighted imaging; FS: Fat saturated; HRCT: High resolution computed tomography; $\mathrm{KOH}$ : Potassium hydroxide: RT-PCR: Reverse transcriptase-polymerase chain reaction; HPE: Histopathological evaluation; LPCB: Lactophenol cotton blue; SDA: Sabouraud dextrose agar

\section{Acknowledgements}

The authors would like to express their deepest gratitude to Sudeep Singh Awal for his technical guidance, post-processing, and image editing.

\section{Authors' contributions}

SSA conceived the study, analyzed and interpreted the radiological studies, drafted the manuscript. SSB participated in the manuscript design and coordination and analyzed and interpreted the radiological studies. SKA participated in the manuscript design and coordination and analyzed and interpreted the microbiological studies. The authors read and approved the final manuscript.

\section{Funding}

Not applicable.

\section{Availability of data and materials}

The data and materials supporting the findings of this study are available on request from the corresponding author.

\section{Declarations}

\section{Ethics approval and consent to participate}

This study was approved by the ethical committee of the institution. Written informed consent was obtained from the patients for publication of this case series and accompanying images.

\section{Consent for publication}

All authors read and approved the final manuscript. Patients included in this research gave written informed consent to publish the data and materials contained within this study.

\section{Competing interests}

The authors declare that they have no competing interests.

\section{Author details}

'Department of Radiology, Jeevandeep Diagnostics, Jamshedpur, India. 2Department of Radiology, Radiance Teleradiology Services, Navi Mumbai, India. ${ }^{3}$ Department of Microbiology, Guru Gobind Singh Medical College \& Hospital, Faridkot, Punjab, India.

Received: 29 May 2021 Accepted: 11 June 2021

Published online: 22 June 2021

\section{References}

1. World Health Organization. Coronavirus. https://www.who.int/health-topics/ coronavirus\#tab=tab_1. Accessed 22 May 2021.

2. World Health Organization. Coronavirus. https://www.who.int/docs/defaultsource/coronaviruse/situation-reports/20200423-sitrep-94-covid-19.pdf. Accessed 22 May 2021

3. Mohan BS, Nambiar V (2020) COVID-19: an insight into SARS-CoV-2 pandemic originated at Wuhan City in Hubei Province of China. J Infect Dis Epidemiol 6:146. https://doi.org/10.23937/2474-3658/1510146

4. Kwee TC, Kwee RM (2020) Chest CT in COVID-19: what the radiologist needs to know. RadioGraphics 40(7):1848-1865. https://doi.org/10.1148/ rg.2020200159

5. Mehta S, Pandey A (2020) Rhino-orbital mucormycosis associated with COVID-19. Cureus 12:e10726. https://doi.org/10.7759/cureus.10726

6. Dyer O (2021) Covid-19: India sees record deaths as "black fungus" spreads fear. BMJ 373. https://doi.org/10.1136/bmj.n1238

7. Ferguson BJ (2000) Mucormycosis of the nose and paranasal sinuses. Otolaryngol Clin North Am. 33(2):349-365. https://doi.org/10.1016/s00306665(00)80010-9 PMID: 10736409

8. Centers for Disease Control and Prevention. Mucormycosis statistics. https:// www.cdc.gov/fungal/diseases/mucormycosis/statistics.html Page last reviewed: May 27, 2020. Accessed 22 May 2021.
9. Kanne JP, Little BP, Chung JH, Elicker BM, Ketai LH (2020) Essentials for radiologists on COVID-19: an update-radiology scientific expert panel. Radiology 296(2):E113-E114. https://doi.org/10.1148/radiol.2020200527

10. Taylor AM, Vasan K, Wong EH, Singh N, Smith M, Riffat F, Sritharan N (2020) Black Turbinate sign: MRI finding in acute invasive fungal sinusitis. Otolaryngol Case Rep 17:100222, ISSN 2468-5488. https://doi.org/10.1016/j. xocr.2020.100222

11. Safder S, Safder CJ, Safder RT, Safder BN, Safder. The "Black Turbinate" sign: an early MR imaging finding of nasal mucormycosis. (2010) Am J Neuroradiol. doi: https://doi.org/10.3174/ajnr.A1808

12. Bala K, Chander J, Handa U, Punia RS, Attri AK (2015) A prospective study of mucormycosis in north India: experience from a tertiary care hospital. Med Mycol 53(3):248-257. https://doi.org/10.1093/mmy/myu086

13. Hallinan JT, Pillay P, Koh LH, Goh KY, Yu WY (2016) Eye globe abnormalities on MR and CT in adults: an anatomical approach. Korean J Radiol 17(5):664673. https://doi.org/10.3348/kjr.2016.17.5.664

14. Chen X, Liao B, Cheng L, Peng X, Xu X, Li Y, Hu T, Li J, Zhou X, Ren B (2020) The microbial coinfection in COVID-19. Appl Microbiol Biotechnol 104(18): 7777-7785. https://doi.org/10.1007/s00253-020-10814-6

15. Werthman-Ehrenreich A (2021) Mucormycosis with orbital compartment syndrome in a patient with COVID-19. Am J Emerg Med 42:264.e5-264.e8, ISSN 0735-6757. https://doi.org/10.1016/j.ajem.2020.09.032

16. Prakash H, Chakrabarti A (2019) Global epidemiology of mucormycosis. J Fungi (Basel) 5(1):26. https://doi.org/10.3390/jof5010026 PMID: 30901907; PMCID: PMC6462913

17. Herrera, D. A., Dublin, A. B., Ormsby, E. L., Aminpour, S., \& Howell, L. P. (2009). Imaging findings of rhinocerebral mucormycosis. Skull base: official journal of North American Skull Base Society ... [et al.], 19(2), 117-125. doi: https://doi.org/10.1055/s-0028-1096209

18. Therakathu J, Prabhu S, Irodi A, Sudhakar SV, Yadav VK, Rupa V (2018) Imaging features of rhinocerebral mucormycosis: a study of 43 patients. Egypt J Radiol Nuclear Med 49(2):447-452. https://doi.org/10.1016/j.ejrnm.2 018.01 .001

\section{Publisher's Note}

Springer Nature remains neutral with regard to jurisdictional claims in published maps and institutional affiliations.

\section{Submit your manuscript to a SpringerOpen ${ }^{\circ}$ journal and benefit from:}

- Convenient online submission

- Rigorous peer review

- Open access: articles freely available online

High visibility within the field

- Retaining the copyright to your article

Submit your next manuscript at $\boldsymbol{\nabla}$ springeropen.com 